\title{
Differential Effects of Hip Rotation Range on Knee Abduction Biomechanics during Double-Legged Landing between Males and Females
}

\author{
Tadashi Yasuda ${ }^{1,2^{*}}$, Keiichi Oyanagi ${ }^{2}$, Miyu Nakagaki ${ }^{2}$, Hiromitsu Itoh $^{3}$ \\ ${ }^{1}$ Department of Orthopaedic Surgery, Kobe City Medical Center General Hospital, Japan \\ ${ }^{2}$ Department of Rehabilitation, Kobe City Medical Center General Hospital, Japan \\ ${ }^{3}$ Department of Physical Therapy, Faculty of Nursing and Rehabilitation, Konan Women's University, Japan
}

\begin{abstract}
Received: July 20, 2020

Accepted: October 15, 2020

Published online: October 31, 2020

Keywords:

Hip Rotation

Kinematics

Kinetics

Knee Joint

Landing Biomechanics
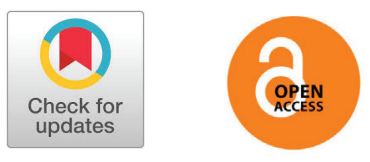

OBJECTIVES Dynamic knee valgus is composed of hip-knee coupling. While females differ from males in passive hip motion, hip rotation range may alter muscle mechanics and neuromuscular activity. This study aimed to compare knee abduction biomechanics during double-legged drop-landing between males and females with different hip rotation ranges.

METHODS This study included five females with the range of hip internal rotation (IR) $>$ the range of hip external rotation (ER), five females with $E R>I R$, four males with $I R>E R$, and five males with $E R>I R$. There was no difference in other hip motions among them or no difference in hip muscle strength between the same sex groups. Three-dimensional motion analyses of the hip and knee joints were performed during double-legged drop-landing.

RESULTS Multiple regression analysis of females with IR $>$ ER showed that peak knee abduction moment (KAM) was associated with maximal hip abduction moment before detecting peak KAM whereas peak knee abduction angle (KAA) correlated with no variable. In females with $E R>I R$, peak KAM was associated with maximal hip ER moment before detecting peak KAM, hip ER muscle strength and hip adduction range while peak KAA correlated with peak hip abduction moment before detecting peak KAM. In males with IR>ER, peak KAM was associated with hip ER range and hip adductor strength whereas peak KAA correlated with maximal hip ER moment and maximal hip IR angle during landing. In males with $E R>I R$, peak KAM was associated with hip extensor strength, hip abduction range and hip flexion range whereas peak KAA correlated with hip ER moment before detecting peak KAM, hip ER muscle strength, and hip adduction range.

CONCLUSIONS Hip rotation range may differentially affect hip-knee coupling strategy for knee abduction control during double-legged drop-landing between males and females.
\end{abstract}

(C) The Asian Society of Kinesiology and the Korean Academy of Kinesiology

\section{Introduction}

Compared with males, females suffer a non-contact anterior cruciate ligament (ACL) injury during sports at a

*Correspondence: Tadashi Yasuda, Department of Orthopaedic Surgery, Kobe City Medical Center General Hospital, 2-1-1 Minatojima-minamimachi, Chuo-ku, Kobe 650-0047, Japan; Tel: +81-78-302-4321; Fax: +81-78-302-7537; E-mail: tadyasu@kcho.jp higher incidence [1]. Greater peak knee abduction moments (KAM) and peak knee abduction angles (KAA) during doublelegged drop-vertical jump tasks predict ACL injury with high specificity and sensitivity in female athletes [2]. Previous studies have focused specifically on the knee joint to investigate sex differences in lower-extremity neuromuscular control and biomechanics [3, 4]. Recent biomechanical, epidemiological, 
and neuromuscular studies indicate that hip functions may affect knee loading $[5,6]$. The kinetic chain consisting of the trunk, hips, knees, and ankles controls lower-extremity movements in a proximal-to-distal sequence [7]. Indeed, hip rotation moment of the uninvolved limb could predict a second ACL injury after ACL reconstruction [8]. Thus, it is important to consider the effects of the hip on the control of the knee. However, the link between proximal control and abnormal knee-joint loading and motions specifically related to sex differences is still under investigation.

Dynamic knee valgus comprises hip internal rotation (IR), hip adduction, knee abduction and tibial external rotation, and plays a critical role in non-contact ACL injury [9]. Currently, factors leading to dynamic knee valgus remain unclarified. Dynamic knee valgus is composed of hip-knee coupling in the frontal and transverse planes and movement occurring in a proximal-to-distal pattern [10]. Therefore, control at the hip may be key to control the knee. In addition, dynamic knee valgus is potentially associated with passive hip range of motion (ROM) [11]. The lower extremity alignments in combination with the lower kinetic chain that structurally position the lower extremity in a more inwardly rotated hip and valgus knee position may contribute to dynamic alignments and increased moments leading to ACL injury. Hip muscle strength may also participate in frontal-plane knee control during dynamic movements [12]. Biomechanically, the hip abductors and hip external rotators (ER-ors) likely prevent knee valgus that results from hip adduction and IR when the knee is loaded under weight-bearing conditions [13].

Sex difference in knee biomechanics during cutting and landing activities has been studied extensively. Compared with males, females likely display greater dynamic knee valgus during those activities [9]. Females differ from males in passive hip ROM [14]. Ranges of hip IR and external rotation (ER) are highly correlated with radiographic measures of femoral anteversion [15]. Females show greater hip IR-ROM than males regardless of sports [16]. Greater femoral anteversion is linked to greater knee ER motion during a controlled single-legged squat [17]. Furthermore, femoral anteversion could alter muscle mechanics and neuromuscular activity during hip abduction and ER $[18,19]$. Sex differences in ACL injury rates emerge after the onset of puberty [20]. While no significant difference in femoral anteversion is found between sexes before the onset of puberty [21,22], femoral anteversion becomes greater in females than in males after the onset of puberty [23]. The sex difference in hip anatomy may in part explain why females demonstrate dynamic knee valgus and an increased risk of ACL injury. However, there has been no study that stratifies the hip-knee coupling by hip rotation ROM associated with femoral anteversion. Thus, this study aimed to examine males and females separately based on their hip rotation range and compare knee abduction biomechanics during double-legged drop-landing. We expected sex difference in knee abduction biomechanics during the landing between sexes with different hip rotation range.

\section{Methods}

\section{Participants}

In this study, young healthy volunteers were classified into four groups based on the hip rotation measurement and sex. The participants had no medical concerns that could potentially effect lower extremity biomechanics or no medical or neurological condition that would impair their ability to perform the experimental task. Group 1 included females who showed that hip IR-ROM was greater than ER-ROM (IR>ER). Group 2 included females who exhibited that hip ER-ROM was greater than IR-ROM (ER $>$ IR). While group 3 included males with IR $>E R$, group 4 included males with ER $>$ IR. Assuming a level of $0.05,80 \%$ power and large effect size (f) of 0.40 [24], a priori analysis with $G^{\star}$ power software demonstrated that 19 people would be needed in each group. At the beginning of this study, we attempted to recruit the required number of groups 2 and 3 because females are more likely to display IR $>$ ER whereas males are more likely to exhibit ER>IR [16]. Eventually, we recruited five females with ER $>$ IR (group 2) and five males with IR $>$ ER (group 3). We excluded one male with IR $>$ ER because of extremely low strength of hip muscles compared with four other males with IR $>$ ER. Then, we recruited five females with IR $>$ ER (group 1) and five males with ER $>$ IR (group 4) after matching the same sex counterparts in relationship to age, height, body 
weight, body mass index, hip muscle strength, and hip and knee ROM except IR and ER < Table 1>. Based on the previous study [25], all data were collected from right lower extremity, which was the dominant leg of each participant defined as the preferred leg for kicking a ball. This study was approved by the Institutional Review Board of our institute (acknowledgement number: n160302).

\section{Measurement of Joint Motion}

Six motions of hip joint (flexion, extension, abduction, adduction, ER, and IR) and two motions of knee joint (flexion and extension) were assessed with the standard procedure using a goniometer [26]. We summed IR-ROM and ER-ROM to calculate IR+ER and subtracted ER-ROM from IR-ROM to calculate IR-ER. The three measurements taken for each ROM of the right leg were averaged for analysis. Our preliminary test for intra-rater reliability by a single examiner (K.O.) revealed that the intra-class correlation coefficient (ICC) $(1,3)$ for same-session data of three trials and ICC $(1,2)$ for betweenday data of two sessions separated by a two-day interval ranged from 0.94 to 0.99 for each ROM measurement of five volunteers.

\section{Measurement of Hip Muscle Strength}

According to a standardized procedure using a handheld dynamometer with a reported high degree of reliability and no systematic test-retest bias [27], hip muscle strength of internal rotator (IR-or), ER-or, adductor, abductor and extensor were evaluated using the hand-held dynamometer, $\mu$ Tas F-1 (Anima Inc. Chofu, Japan), for isometric strength measurement. With neutral position of the knee and hip in frontal and transverse planes in the sitting position, the force pad of the hand-held dynamometer was placed on the medial or lateral malleolus of the ankle for ER-or or IR-or measurement and placed on the lateral or medial epicondyle of the femur for hip abductor or adductor strength measurement. Isometric hip extensor strength was also measured using the hand-held dynamometer placed on the posterior aspect of distal femur with the participants in the prone position, and the hip in neutral position. Those muscle strengths of the dominant leg were measured in a randomized order to avoid systematic bias. The participant exerted a 5-second maximum isometric contraction against the hand-held dynamometer. The test was performed twice. Between the two test trials, a rest period of one minute was introduced. The highest force value during the two consecutive measurements was used for data analysis. Hip muscle strength of extensor, abductor and adductor was expressed as the maximal voluntary force per $\mathrm{kg}$ body weight. Muscle strength of ER-or and IR-or was shown as the torque, calculated using the lever arm length from the medial knee epicondyle to the center of the dynamometer, per $\mathrm{kg}$ body weight. Our preliminary test for intra-rater reliability by a single examiner (M.N.) showed that ICC $(1,1)$ for samesession data of two trials and ICC $(1,1)$ for between-day data of two sessions separated by a two-day interval ranged from 0.93-0.96 for measurement of maximal isometric strength of hip muscles of five volunteers.

\section{Three-Dimensional Motion Analysis of the Hip and Knee during Double-Legged Drop Landing}

A three-dimensional motion analysis of the hip and knee during double-legged drop landing was performed using a MAC3D automatic digitizing motion analysis system with eight cameras (Motion Analysis Corporation, Santa Rosa, CA, USA) and force plate (AMTI, Watertown, Massachusetts, USA). The sampling rates were set at $200 \mathrm{~Hz}$ for camera data and at $1000 \mathrm{~Hz}$ for force data. We chose natural double-legged drop-landing $[28,29]$ for analysis. For the preferred natural condition, drop landings were initiated from a $30 \mathrm{~cm}$ tall box positioned directly behind the force plate. Participants were instructed to reach out with their dominant foot to position it over the force plate; roll forward off of the box using their nondominant foot without jumping or lowering themselves to initiate a drop; and then to perform a double-leg terminal landing with their dominant foot positioned on the force plate and their nondominant foot positioned on the floor next to the force plate.

For assessment of the drop landing motion, the IOR (Istituto Orthopedico Rizzoli) full-body model was applied for definition of bilateral upper- and lower-body segments including the CODA model for the pelvis [30,31]. The locations of the retroreflective markers were 1) head segment, 
2) spinous processes of the seventh cervical vertebra, second and seventh thoracic vertebrae, jugular notch, xiphoid process, and the first, third, and fifth lumbar vertebrae, 3) acromion, deltoid tuberosity, lateral humeral epicondyle, dorsal forearm, styloid processes of the ulna and radius and head of the third metacarpal, 4) anterior superior and posterior superior iliac spines, 5) the greater trochanter, the lateral and medial femoral epicondyles, 6) the fibular head, the tibial tuberosity, the lateral and medial malleoli, and 7) proximal posterior surface of the calcaneus and distal attachment of the Achilles tendon on the calcaneus, the sustentaculum tali, the peroneal tubercle, the navicular tuberosity, the first, second and fifth metatarsal heads, proximal distal phalanx, bases of the first and second metatarsals, and styloid process of the fifth metatarsal [31]. A series of calibration trials in anatomical standing posture was performed to determine anatomical landmarks and lower limb joint coordinate systems [31]. After two practice trials, each participant performed three trials of the drop-landing task. The landing phase was defined as the period between the point of initial ground contact (IGC) and the peak knee flexion angle. IGC was defined as when the vertical ground reaction force was greater than $10 \mathrm{~N}$ [25]. Peak knee valgus during landing generally occur less than $150 \mathrm{~ms}$ after IGC $[32,33]$. Thus, we focused on the motion analysis data during $150 \mathrm{~ms}$ after IGC.

Tracking the motions of the markers, sampling, interpolation of missing data, and filtering the raw threedimensional coordinate data were performed as described previously [31]. The joint angles and moments were calculated using the Euler angle method for hip flexion/extension, adduction/abduction, and IR/ER sequences according to the recommendations of the International Society of Biomechanics [34].

For data analysis of the kinematic and kinetic parameters of the hip and knee, angle and moment values for hip flexion/ extension, adduction/abduction and IR/ER were calculated, in which flexion, adduction and IR were denoted as positive. The parameters adopted for the analysis were the angle and moment average values of the three trials. The moment values were normalized by body weight. Analytical parameters derived from clinical measurement of the hip were flexion/ extension, adduction/abduction and IR/ER angles for ROM and peak joint angle, and the weight-adjusted extensor and abductor/adductor forces and ER-or/IR-or torque values for strength.

\section{Statistical Analysis}

The data are expressed as the mean \pm standard deviation. After Shapiro-Wilk test of normality, the data were compared between four groups using a one-way analysis of variance for parametric data or Kruskal-Wallis test for non-parametric data accompanied by a post hoc Bonferroni correction for multiple comparisons with an adjusted a level set $a$ priori at 0.0125. Cohen's d was calculated for the comparison between two means. Pearson's correlation coefficients were calculated to assess the relationship between kinematic/kinetic variables of the knee and the parameters of the hip. Multiple regression analysis was performed using stepwise method. We judged multicollinearity by variance inflation factor. The significance level was set at $<0.05$. Statistical analyses were performed using the SPSS (version 25.0, IBM, Chicago, IL, USA).

\section{Results}

\section{Comparison of Demographics <Table 1>}

Group 1 females and group 3 males showed greater IRROM and IR-ER compared with group 2 females and group 4 males, respectively. Group 4 exhibited greater ER-ROM than group 3. There was no difference in IR+ER or other demographics between groups 1 and 2 or between groups 3 and 4. Group 4 displayed stronger hip extensor than females. The males exhibited stronger hip adductor than group 2 . While the males showed stronger ER-or than group 1, group 3 exhibited stronger ER-or than group 2.

\section{Comparison of Data from Motion Analysis <Table 2>}

Peak KAM and peak KAA were selected as dependent variables for multiple regression analysis described below. There was no difference in peak KAM or peak KAA between groups, although the males tended to show greater peak KAA than the females.

The females likely exhibited smaller maximal hip flexion, abduction, and IR angles during 150 ms after IGC compared 
Table 1. Comparison of demographic data

\begin{tabular}{|c|c|c|c|c|c|c|c|c|c|c|c|}
\hline \multirow{3}{*}{$\begin{array}{l}\text { Sex } \\
\text { Group }\end{array}$} & \multicolumn{2}{|c|}{ Female } & \multicolumn{2}{|c|}{ Male } & \multirow{3}{*}{$P^{* *}$} & \multicolumn{6}{|c|}{$P$ by post-hoc analysis between groups } \\
\hline & \multirow{2}{*}{$\frac{1}{\operatorname{IR}>\operatorname{ER}(n=5)}$} & \multirow{2}{*}{$\frac{2}{E R>I R(n=5)}$} & \multirow{2}{*}{$\frac{3}{\operatorname{IR}>\operatorname{ER}(n=4)}$} & \multirow{2}{*}{$\frac{4}{E R>I R(n=5)}$} & & \multicolumn{6}{|c|}{ (Cohen's d) } \\
\hline & & & & & & 1 and 2 & 1 and 3 & 1 and 4 & 2 and 3 & 2 and 4 & 3 and 4 \\
\hline Age (years) & $23.2 \pm 0.8$ & $24.6 \pm 1.1$ & $19.0 \pm 4.0^{*}$ & $17.7 \pm 0.2$ & 0.015 & $1.000(1.40)$ & $0.033(1.56)$ & $0.003(9.08)$ & $0.004(2.03)$ & $<0.001(8.43)$ & $1.000(0.48)$ \\
\hline Height (cm) & $157 \pm 4.3$ & $159 \pm 2.8$ & $171 \pm 2.4$ & $172 \pm 3.4$ & $<0.001$ & $1.000(0.67)$ & $<0.001(3.85)$ & $<0.001(3.97)$ & $0.001(4.36)$ & $<0.001(4.15)$ & $1.000(0.51)$ \\
\hline Weight (kg) & $49.2 \pm 2.9$ & $51.1 \pm 5.2$ & $58.1 \pm 2.2$ & $58.0 \pm 5.3$ & 0.009 & $1.000(0.45)$ & $0.042(3.37)$ & $0.031(2.06)$ & $0.159(1.66)$ & $0.127(1.31)$ & $1.000(0.02)$ \\
\hline $\begin{array}{l}\text { Body mass } \\
\text { index }\left(\mathrm{kg} / \mathrm{m}^{2}\right)\end{array}$ & $20.0 \pm 1.9$ & $20.1 \pm 1.5$ & $19.9 \pm 1.2$ & $19.7 \pm 1.4$ & 0.909 & $1.000(0.02)$ & $1.000(0.08)$ & $1.000(0.37)$ & $1.000(0.12)$ & $1.000(0.46)$ & $1.000(0.38)$ \\
\hline \multicolumn{12}{|l|}{ Hip motion $\left({ }^{\circ}\right)$} \\
\hline Flexion & $143 \pm 7.1$ & $139 \pm 13.0$ & $125 \pm 8.8$ & $136 \pm 5.9$ & 0.066 & $1.000(0.31)$ & $0.075(2.17)$ & $1.000(1.04)$ & $0.221(1.28)$ & $1.000(0.36)$ & $0.630(1.40)$ \\
\hline Extension & $19.4 \pm 1.3^{*}$ & $21.6 \pm 6.5$ & $32.3 \pm 10.6$ & $31.4 \pm 9.9^{*}$ & 0.063 & $1.000(0.47)$ & $0.157(1.71)$ & $0.164(1.69)$ & $0.353(1.22)$ & $0.387(1.17)$ & $1.000(0.08)$ \\
\hline Adduction & $9.0 \pm 1.4^{*}$ & $15.4 \pm 4.5$ & $18.5 \pm 9.0$ & $20.0 \pm 7.1$ & 0.024 & $0.660(1.92)$ & $0.187(1.48)$ & $0.063(2.15)$ & $1.000(0.44)$ & $1.000(0.77)$ & $1.000(0.19)$ \\
\hline Abduction & $45.2 \pm 8.0$ & $51.4 \pm 8.2$ & $45.5 \pm 2.6$ & $50.0 \pm 13.2$ & 0.644 & $1.000(0.77)$ & $1.000(0.05)$ & $1.000(0.44)$ & $1.000(0.97)$ & $1.000(0.13)$ & $1.000(0.47)$ \\
\hline IR & $67.8 \pm 4.7$ & $37.6 \pm 12.9^{*}$ & $48.0 \pm 5.0$ & $29.0 \pm 10.0$ & 0.003 & $0.001(3.11)$ & $0.032(4.08)$ & $<0.001(4.96)$ & $0.646(1.06)$ & $0.926(0.74)$ & $0.042(2.40)$ \\
\hline ER & $45.0 \pm 12.7$ & $52.8 \pm 15.8$ & $36.5 \pm 7.9$ & $70.4 \pm 10.4$ & 0.005 & $1.000(0.55)$ & $1.000(0.80)$ & $0.031(2.19)$ & $0.400(1.30)$ & $0.233(1.32)$ & $0.006(3.66)$ \\
\hline $\mathrm{IR}+\mathrm{ER}$ & $113 \pm 16.8$ & $90.4 \pm 28.5^{*}$ & $84.5 \pm 10.7$ & $99.4 \pm 15.6$ & 0.129 & $0.536(0.96)$ & $0.282(2.01)$ & $1.000(0.83)$ & $1.000(0.27)$ & $1.000(0.39)$ & $1.000(1.11)$ \\
\hline IR-ER & $22.8 \pm 9.0$ & $-15.2 \pm 4.6$ & $11.5 \pm 7.9$ & $-41.4 \pm 13.2$ & $<0.001$ & $<0.001(5.31)$ & $0.539(1.33)$ & $<0.001(5.68)$ & $0.004(4.13)$ & $0.003(2.66)$ & $<0.001(4.86)$ \\
\hline \multicolumn{12}{|l|}{ Knee motion $\left(^{\circ}\right)$} \\
\hline Flexion & $149 \pm 3.8$ & $155 \pm 3.4$ & $149 \pm 2.1$ & $152 \pm 1.3$ & 0.024 & $0.072(1.44)$ & $1.000(0.29)$ & $1.000(0.85)$ & $0.039(2.15)$ & $0.868(1.08)$ & $0.648(1.90)$ \\
\hline Extension & $0.4 \pm 7.0$ & $0.6 \pm 1.3^{*}$ & $7.0 \pm 9.5$ & $0.6 \pm 1.9^{*}$ & 0.420 & $1.000(0.04)$ & $0.630(0.79)$ & $1.000(0.04)$ & $0.690(0.94)$ & $1.000(0.00)$ & $0.690(0.93)$ \\
\hline \multicolumn{12}{|c|}{ Hip muscle strength } \\
\hline Extensor (N/kg) & $6.34 \pm 1.12$ & $6.39 \pm 1.79$ & $7.60 \pm 0.82$ & $10.48 \pm 1.68$ & 0.001 & $1.000(0.03)$ & $1.000(1.29)$ & $0.002(2.90)$ & $1.000(0.88)$ & $0.003(2.36)$ & $0.056(2.18)$ \\
\hline $\begin{array}{l}\text { Adductor } \\
(\mathrm{N} / \mathrm{kg})\end{array}$ & $3.78 \pm 0.60$ & $3.01 \pm 1.27$ & $5.45 \pm 0.47$ & $5.19 \pm 0.57$ & 0.001 & $0.934(0.77)$ & $0.046(3.11)$ & $0.091(2.41)$ & $0.003(2.54)$ & $0.004(2.21)$ & $1.000(0.51)$ \\
\hline $\begin{array}{l}\text { Abductor } \\
\text { (N/kg) }\end{array}$ & $4.59 \pm 1.43$ & $4.43 \pm 1.11$ & $5.86 \pm 0.81$ & $6.81 \pm 1.44$ & 0.028 & $1.000(0.13)$ & $0.904(1.09)$ & $0.080(1.54)$ & $0.643(1.47)$ & $0.052(1.85)$ & $1.000(0.81)$ \\
\hline $\begin{array}{l}\text { Internal rotator } \\
(\mathrm{Nm} / \mathrm{kg})\end{array}$ & $5.85 \pm 1.90$ & $5.56 \pm 0.98$ & $7.37 \pm 1.22$ & $8.07 \pm 3.03$ & 0.196 & $1.000(0.18)$ & $1.000(0.95)$ & $0.589(0.88)$ & $1.000(1.61)$ & $0.401(1.11)$ & $1.000(0.30)$ \\
\hline $\begin{array}{l}\text { External rotator } \\
(\mathrm{Nm} / \mathrm{kg})\end{array}$ & $5.65 \pm 0.67$ & $6.87 \pm 1.50$ & $9.99 \pm 1.34$ & $9.10 \pm 0.78$ & $<0.001$ & $0.629(1.05)$ & $<0.001(4.11)$ & $0.001(4.75)$ & $0.005(2.20)$ & $0.038(1.86)$ & $1.000(0.82)$ \\
\hline
\end{tabular}

with the males whereas the females tended to show greater maximal adduction angle than the males. No difference was found in maximal hip motion during $150 \mathrm{~ms}$ after IGC. There was no difference in maximal hip moment during 150 ms after IGC or before detecting peak KAM between groups. In addition, there was no difference in maximal knee flexion angle during $150 \mathrm{~ms}$ after IGC between groups (data not shown).

\section{Correlations between dependent variables and demographics}

We analyzed correlations of peak KAM and peak KAA with the demographic data in each group $<$ Table $3>$. In group 1, peak KAM correlated negatively with hip adduction-ROM and abduction-ROM. In group 2, peak KAM correlated positively with hip extension-ROM while peak KAA correlated negatively with hip abduction-ROM. In group 3, peak KAM correlated negatively with ER-ROM and IR-or. In group 4, peak KAM correlated positively with hip extensor and ER-or.

\section{Correlations between Dependent Variables and Parameters from Motion Analysis}

We further analyzed correlations of the dependent variables with the parameters from motion analysis in each 
Table 2. Comparison of parameters from motion analysis and dependent variables

\begin{tabular}{|c|c|c|c|c|c|c|c|c|c|c|c|}
\hline \multirow{3}{*}{$\begin{array}{l}\text { Sex } \\
\text { Group }\end{array}$} & \multicolumn{2}{|c|}{ Female } & \multicolumn{2}{|c|}{ Male } & \multirow{3}{*}{$P^{* *}$} & \multicolumn{6}{|c|}{$P$ by post-hoc analysis between groups } \\
\hline & \multirow{2}{*}{$\frac{1}{I R>E R(n=5)}$} & \multirow{2}{*}{$\frac{2}{E R>I R(n=5)}$} & \multirow{2}{*}{$\frac{3}{I R>E R(n=4)}$} & \multirow{2}{*}{$\frac{4}{E R>I R(n=5)}$} & & \multicolumn{6}{|c|}{ (Cohen's d) } \\
\hline & & & & & & 1 and 2 & 1 and 3 & 1 and 4 & 2 and 3 & 2 and 4 & 3 and 4 \\
\hline \multicolumn{12}{|c|}{ Motion analysis parameters } \\
\hline \multicolumn{12}{|c|}{ Maximal hip motion during $150 \mathrm{~ms}$ after IGC $\left(^{\circ}\right)$} \\
\hline Flexion & $45.5 \pm 6.0$ & $43.1 \pm 14.9$ & $66.3 \pm 5.7$ & $59.4 \pm 13.5$ & 0.019 & $1.000(0.21)$ & $0.085(3.56)$ & $0.402(1.33)$ & $0.044(2.05)$ & $0.209(1.15)$ & $1.000(0.66$ \\
\hline Adduction & $-4.0 \pm 4.7$ & $-3.3 \pm 4.0$ & $-12.0 \pm 5.3$ & $-10.2 \pm 3.0^{*}$ & 0.025 & $1.000(0.14)$ & $0.078(1.61)$ & $0.216(1.58)$ & $0.050(1.84)$ & $0.138(1.92)$ & $1.000(0.43$ \\
\hline Abduction & $5.2 \pm 4.1$ & $4.9 \pm 2.9$ & $14.8 \pm 7.1$ & $12.9 \pm 2.6$ & 0.005 & $1.000(0.09)$ & $0.027(1.67)$ & $0.077(2.25)$ & $0.021(1.84)$ & $0.061(2.92)$ & $1.000(0.37$ \\
\hline IR & $2.0 \pm 2.1$ & $0.2 \pm 5.9$ & $1.1 \pm 6.6$ & $3.6 \pm 9.7$ & 0.879 & $1.000(0.39)$ & $1.000(0.17)$ & $1.000(0.23)$ & $1.000(0.14)$ & $1.000(0.42)$ & $1.000(0.30$ \\
\hline ER & $0.7 \pm 2.7$ & $2.7 \pm 6.5$ & $13.0 \pm 6.7$ & $10.2 \pm 7.9$ & 0.027 & $1.000(0.41)$ & $0.062(2.40)$ & $0.182(1.59)$ & $0.164(1.55)$ & $0.480(1.02)$ & $1.000(0.38$ \\
\hline \multicolumn{12}{|c|}{ Maximal hip moment during 150 ms after IGC (Nm) } \\
\hline Abduction moment & $1.78 \pm 1.98$ & $1.69 \pm 1.55^{*}$ & $0.87 \pm 0.52$ & $0.66 \pm 0.35$ & 0.467 & $1.000(0.05)$ & $1.000(0.63)$ & $1.000(0.79)$ & $1.000(0.72)$ & $1.000(0.92)$ & $1.000(0.48$ \\
\hline $\begin{array}{l}\text { External rotation } \\
\text { moment }\end{array}$ & $0.76 \pm 0.68^{*}$ & $0.83 \pm 0.81$ & $0.41 \pm 0.12$ & $0.32 \pm 0.18$ & 0.926 & $1.000(0.09)$ & $1.000(0.73)$ & $1.000(0.89)$ & $1.000(0.73)$ & $1.000(0.86)$ & $1.000(0.54$ \\
\hline \multicolumn{12}{|c|}{ Maximal hip moment before detecting peak KAM (Nm) } \\
\hline Abduction moment & $1.78 \pm 1.98$ & $0.63 \pm 0.75$ & $0.79 \pm 0.42$ & $0.66 \pm 0.35$ & 0.346 & $0.750(0.77)$ & $1.000(0.69)$ & $0.798(0.79)$ & $1.000(0.26)$ & $1.000(0.04)$ & $1.000(0.35$ \\
\hline $\begin{array}{l}\text { External rotation } \\
\text { moment }\end{array}$ & $0.70 \pm 0.74$ & $0.13 \pm 0.43^{*}$ & $0.34 \pm 0.21$ & $0.30 \pm 0.16$ & 0.241 & $0.433(0.93)$ & $1.000(0.65)$ & $1.000(0.74)$ & $1.000(0.62)$ & $1.000(0.51)$ & $1.000(0.24$ \\
\hline \multicolumn{12}{|l|}{ Dependent variables } \\
\hline peak KAM (Nm) & $1.80 \pm 1.90$ & $1.12 \pm 1.76^{*}$ & $0.50 \pm 0.32$ & $0.64 \pm 0.20$ & 0.687 & $1.000(0.37)$ & $0.836(0.95)$ & $1.000(0.85)$ & $1.000(0.48)$ & $1.000(0.38)$ & $1.000(0.52$ \\
\hline peak KAA $\left({ }^{\circ}\right)$ & $2.42 \pm 2.34$ & $2.15 \pm 6.44$ & $7.47 \pm 2.67$ & $9.92 \pm 3.49^{*}$ & 0.010 & $1.000(0.06)$ & $0.538(2.01)$ & $0.072(2.52)$ & $0.451(1.08)$ & $0.058(1.50)$ & $1.000(0.79$ \\
\hline
\end{tabular}

Data are expressed as mean \pm standard deviation. IR, internal rotation; ER, external rotation; IGC, initial ground contact; KAM, knee abduction moment; KAA, knee abduction angle. ${ }^{*} P<0.05$ by Shapiro-Wilk test. ${ }^{*} P$ values are determined by one-way analysis of variance for parametric data or by KruskalWallis test for non-parametric data.

Table 3. Correlations of dependent variables with demographics

Group 1. Female IR>ER

\begin{tabular}{|c|c|c|c|c|c|c|c|c|c|c|c|c|c|c|}
\hline & \multirow[b]{2}{*}{ BMI } & \multicolumn{8}{|c|}{ Hip range of motion } & \multicolumn{5}{|c|}{ Hip muscle strength } \\
\hline & & Flexion & Extension & Adduction & Abduction & $\mathbb{R}$ & ER & $\mathbb{R}+E R$ & $\mathbb{R}$-ER & Extensor & Abductor & Adductor & IR-or & ER-or \\
\hline peak KAM & 0.801 & -0.407 & -0.515 & -0.929 & -0.830 & -0.588 & -0.139 & -0.268 & -0.108 & 0.260 & 0.115 & 0.004 & -0.683 & 0.112 \\
\hline peak KAA & -0.446 & -0.414 & -0.145 & -0.181 & -0.080 & -0.035 & 0.156 & 0.107 & -0.236 & 0.437 & -0.533 & -0.160 & -0.104 & -0.472 \\
\hline
\end{tabular}

Group 2. Female ER>IR

\begin{tabular}{|c|c|c|c|c|c|c|c|c|c|c|c|c|c|c|}
\hline & \multirow[b]{2}{*}{ BMI } & \multicolumn{8}{|c|}{ Hip range of motion } & \multicolumn{5}{|c|}{ Hip muscle strength } \\
\hline & & Flexion & Extension & Adduction & Abduction & $\mathbb{R}$ & ER & IR+ER & IR-ER & Extensor & Abductor & Adductor & $\mathbb{R}$-or & ER-or \\
\hline peak KAM & -0.039 & -0.105 & 0.833 & -0.271 & -0.281 & -0.104 & -0.243 & -0.182 & 0.550 & -0.580 & 0.045 & 0.187 & 0.078 & 0.270 \\
\hline peak KAA & -0.113 & 0.083 & 0.550 & 0.360 & -0.828 & -0.455 & -0.457 & -0.459 & 0.297 & -0.410 & 0.033 & 0.004 & -0.046 & -0.368 \\
\hline
\end{tabular}

Group 3. Male IR>ER

\begin{tabular}{|c|c|c|c|c|c|c|c|c|c|c|c|c|c|c|}
\hline & \multirow[b]{2}{*}{ BMI } & \multicolumn{8}{|c|}{ Hip range of motion } & \multicolumn{5}{|c|}{ Hip muscle strength } \\
\hline & & Flexion & Extension & Adduction & Abduction & $\mathbb{R}$ & ER & $\mathbb{R}+E R$ & IR-ER & Extensor & Abductor & Adductor & IR-or & ER-or \\
\hline peak KAM & -0.272 & 0.878 & -0.254 & -0.014 & 0.141 & -0.244 & -0.997 & -0.857 & 0.842 & 0.118 & -0.757 & 0.102 & -0.963 & -0.424 \\
\hline peak KAA & -0.637 & 0.477 & -0.238 & -0.894 & -0.064 & 0.551 & 0.087 & 0.325 & 0.262 & 0.139 & 0.664 & 0.799 & -0.001 & 0.784 \\
\hline
\end{tabular}

Group 4. Male ER>IR

\begin{tabular}{|c|c|c|c|c|c|c|c|c|c|c|c|c|c|c|}
\hline & \multirow[b]{2}{*}{ BMI } & \multicolumn{8}{|c|}{ Hip range of motion } & \multicolumn{5}{|c|}{ Hip muscle strength } \\
\hline & & Flexion & Extension & Adduction & Abduction & $\mathbb{R}$ & ER & $\mathrm{IR}+\mathrm{ER}$ & IR-ER & Extensor & Abductor & Adductor & IR-or & ER-or \\
\hline peak KAM & -0.009 & 0.200 & -0.331 & 0.083 & -0.092 & 0.077 & 0.136 & 0.140 & -0.049 & 0.895 & 0.035 & 0.168 & 0.198 & 0.864 \\
\hline peak KAA & -0.760 & 0.782 & 0.069 & 0.741 & 0.191 & 0.478 & 0.567 & 0.685 & -0.085 & -0.146 & -0.160 & -0.480 & 0.215 & 0.396 \\
\hline
\end{tabular}

Pearson's correlation coefficient between peak KAM and IR-ER. Values highlighted in bold indicate statistical significance $(P<0.05)$. KAM, knee abduction moment; KAA, knee abduction angle; BMI, body mass index; IR, internal rotation; ER, external rotation; IR-or, internal rotator; ER-or, external rotator. 
Table 4. Correlations of dependent variables with parameters from motion analysis

Group 1. Female IR>ER

\begin{tabular}{|c|c|c|c|c|c|c|c|c|c|}
\hline & \multicolumn{5}{|c|}{ Maximal hip motion during $150 \mathrm{~ms}$ after IGC } & \multicolumn{2}{|c|}{$\begin{array}{l}\text { Maximal hip moment during } \\
150 \mathrm{~ms} \text { after IGC }\end{array}$} & \multicolumn{2}{|c|}{$\begin{array}{l}\text { Maximal hip moment before } \\
\text { detecting peak KAM }\end{array}$} \\
\hline & Flexion & Abduction & Adduction & ER & IR & Abduction & ER & Abduction & ER \\
\hline peak KAM & 0.301 & -0.925 & 0.947 & 0.371 & -0.556 & 0.998 & 0.955 & 0.998 & 0.962 \\
\hline peak KAA & 0.251 & -0.118 & 0.065 & -0.422 & 0.279 & 0.117 & 0.185 & 0.117 & 0.101 \\
\hline
\end{tabular}

Group 2. Female ER>IR

\begin{tabular}{|c|c|c|c|c|c|c|c|c|c|}
\hline \multirow[b]{3}{*}{ peak KAM } & \multicolumn{5}{|c|}{ Maximal hip motion during 150 ms after IGC } & \multicolumn{2}{|c|}{$\begin{array}{l}\text { Maximal hip moment during } \\
150 \mathrm{~ms} \text { after IGC }\end{array}$} & \multicolumn{2}{|c|}{$\begin{array}{c}\text { Maximal hip moment before } \\
\text { detecting peak KAM }\end{array}$} \\
\hline & Flexion & Abduction & Adduction & ER & IR & Abduction & ER & Abduction & ER \\
\hline & -0.568 & -0.013 & -0.108 & 0.562 & -0.665 & 0.372 & 0.100 & 0.763 & 0.982 \\
\hline peak KAA & 0.024 & -0.518 & 0.489 & 0.801 & -0.772 & 0.884 & 0.723 & 0.941 & 0.739 \\
\hline \multicolumn{10}{|c|}{ Group 3. Male IR>ER } \\
\hline & \multicolumn{5}{|c|}{ Maximal hip motion during 150 ms after IGC } & \multicolumn{2}{|c|}{$\begin{array}{l}\text { Maximal hip moment during } \\
150 \text { ms after IGC }\end{array}$} & \multicolumn{2}{|c|}{$\begin{array}{c}\text { Maximal hip moment before } \\
\text { detecting peak KAM }\end{array}$} \\
\hline & Flexion & Abduction & Adduction & ER & IR & Abduction & ER & Abduction & ER \\
\hline peak KAM & -0.263 & 0.643 & -0.742 & 0.882 & -0.716 & 0.370 & 0.169 & 0.394 & 0.722 \\
\hline peak KAA & -0.803 & -0.509 & 0.337 & -0.139 & -0.608 & 0.860 & 0.979 & 0.727 & 0.674 \\
\hline \multicolumn{10}{|c|}{ Group 4. Male ER>IR } \\
\hline & \multicolumn{5}{|c|}{ Maximal hip motion during 150 ms after IGC } & \multicolumn{2}{|c|}{$\begin{array}{l}\text { Maximal hip moment during } \\
150 \text { ms after IGC }\end{array}$} & \multicolumn{2}{|c|}{$\begin{array}{c}\text { Maximal hip moment before } \\
\text { detecting peak KAM }\end{array}$} \\
\hline & Flexion & Abduction & Adduction & ER & IR & Abduction & ER & Abduction & ER \\
\hline peak KAM & -0.297 & 0.012 & 0.173 & 0.276 & -0.263 & 0.489 & 0.124 & 0.489 & 0.155 \\
\hline peak KAA & -0.856 & -0.772 & 0.194 & 0.358 & -0.556 & 0.726 & 0.906 & 0.726 & 0.937 \\
\hline
\end{tabular}

Pearson's correlation coefficient between peak KAM and maximal hip moment during $150 \mathrm{~ms}$ after IGC. Values highlighted in bold indicate statistical significance $(P<0.05)$. KAM, knee abduction moment; KAA, knee abduction angle; ER, external rotation; IR, internal rotation; IGC, initial ground contact.

group < Table 4>. In group 1, peak KAM correlated positively with maximal hip abduction angle during $150 \mathrm{~ms}$ after IGC, and hip abduction moment and ER moment during $150 \mathrm{~ms}$ after IGC as well as before detecting peak KAM. We also found negative correlation between peak KAM and maximal hip abduction moment during $150 \mathrm{~ms}$ after IGC. In group 2, peak KAM correlated positively with maximal ER moment before detecting peak KAM whereas peak KAA correlated positively with maximal hip abduction moment during $150 \mathrm{~ms}$ after IGC as well as before detecting peak KAM. In group 3, peak KAA correlated positively with maximal ER moment during 150 ms after IGC. In group 4, peak KAA correlated positively with maximal ER moment during $150 \mathrm{~ms}$ after IGC as well as before detecting peak KAM, and negatively with maximal hip flexion angle during $150 \mathrm{~ms}$ after IGC.

40 | The Asian Journal of Kinesiology

\section{Multiple Regression Analysis}

Multiple regression analysis was performed for peak KAM and peak KAA as dependent variables using independent variables of the demographic data $<$ Table $3>$ and the parameters from motion analysis <Table $4>$. The results from multiple regression analysis of the females are shown in $<$ Table $5>$. In group 1, an independent predictor for peak KAM was hip abduction moment before detecting peak KAM. No independent predictor was found for peak KAA. In group 2, independent predictors for peak KAM were ER moment before detecting peak KAM, ER-or strength and hip adduction-ROM. An independent predictor for peak KAA was hip abduction moment before detecting peak KAM. The results of multiple regression analysis of the males are shown in $<$ Table 6>. In group 3, independent predictors for peak KAM were ER-ROM and hip adductor strength. Independent predictors for peak KAA were ER moment during $150 \mathrm{~ms}$ 
Table 5. Multiple regression analysis for female participants

Group 1. Female IR>ER

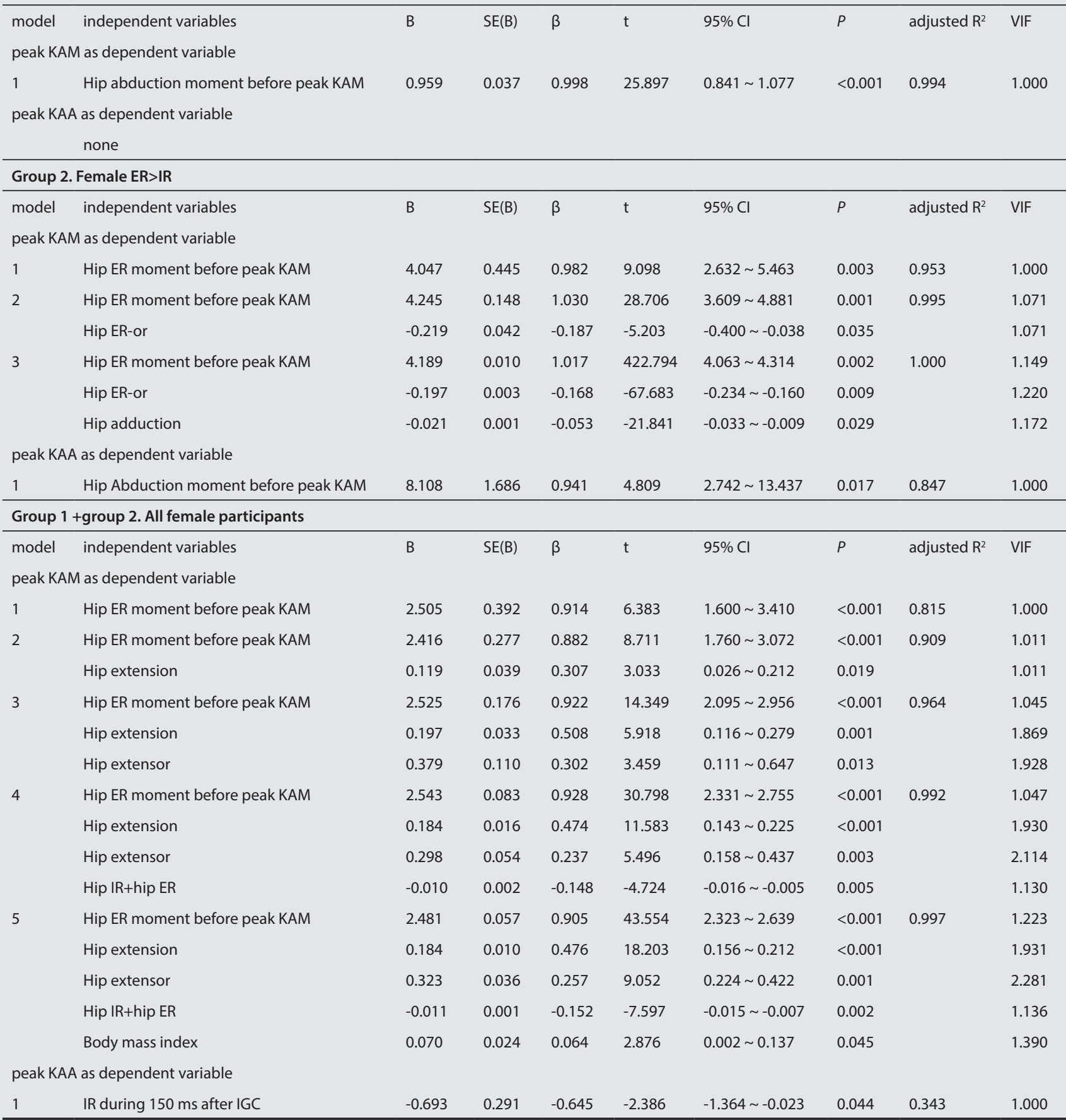

Multiple regression analysis was performed with peak KAM and peak KAA as dependent variables, and with demographics and parameters from motion analysis as independent variables. Selection of modeling was done using stepwise method. B, partial regression coefficient; $\mathrm{SE}$, standard error; $\beta$, standardized partial regression coefficient; $\mathrm{t}, \mathrm{t}$-ratio; $95 \% \mathrm{Cl}, 95 \%$ confidence interval; $P, P$-value; $\mathrm{R}^{2}$, coefficient of determination; $\mathrm{VIF}$, variance inflation factor; IR, internal rotation; ER, external rotation; KAM, knee abduction moment; KAA, knee abduction angle; ER-or, external rotator; IGC, initial ground contact.

after IGC and IR angle during $150 \mathrm{~ms}$ after IGC. In group 4 , independent predictors for peak KAM were hip extensor strength, abduction-ROM, and flexion-ROM. Independent predictors for peak KAA were ER moment before detecting peak KAM, ER-or strength, and hip adduction-ROM.

Multiple regression analysis was also performed using 


\begin{tabular}{|c|c|c|c|c|c|c|c|c|c|}
\hline \multicolumn{10}{|c|}{ Group 3. Male IR>ER } \\
\hline model & independent variables & B & $\mathrm{SE}(\mathrm{B})$ & $\beta$ & $\mathrm{t}$ & $95 \% \mathrm{Cl}$ & $P$ & adjusted $\mathrm{R}^{2}$ & VIF \\
\hline \multicolumn{10}{|c|}{ peak KAM as dependent variable } \\
\hline 1 & Hip ER & -0.040 & 0.002 & -0.997 & -17.423 & $-0.050 \sim-0.030$ & 0.003 & 0.990 & 1.000 \\
\hline \multirow[t]{2}{*}{2} & Hip ER & -0.040 & 0.000 & -9.995 & -3812.2 & $-0.041 \sim-0.040$ & $<0.001$ & 1.000 & 1.000 \\
\hline & Hip adductor & 0.055 & 0.000 & 0.081 & 310.02 & $0.053 \sim 0.058$ & 0.002 & & 1.000 \\
\hline \multicolumn{10}{|c|}{ peak KAA as dependent variable } \\
\hline 1 & Hip ER moment during 150 ms after IGC & 21.597 & 3.171 & 0.979 & 6.811 & $7.954 \sim 35.240$ & 0.021 & 0.938 & 1.000 \\
\hline \multirow[t]{2}{*}{2} & Hip ER moment during 150 ms after IGC & 26.778 & 0.409 & 1.214 & 65.410 & $21.576 \sim 31.980$ & 0.010 & 1.000 & 2.340 \\
\hline & Hip IR during 150 ms after IGC & 0.126 & 0.008 & 0.310 & 16.724 & $0.030 \sim 0.221$ & 0.038 & & 2.340 \\
\hline \multicolumn{10}{|c|}{ Group 4. Male ER>IR } \\
\hline model & independent variables & B & $\mathrm{SE}(\mathrm{B})$ & $\beta$ & $\mathrm{t}$ & $95 \% \mathrm{Cl}$ & $P$ & adjusted $R^{2}$ & VIF \\
\hline \multicolumn{10}{|c|}{ peak KAM as dependent variable } \\
\hline 1 & Hip extensor & 0.108 & 0.031 & 0.895 & 3.481 & $0.009 \sim 0.207$ & 0.040 & 0.735 & 1.000 \\
\hline \multirow[t]{2}{*}{2} & Hip extensor & 0.139 & 0.011 & 1.155 & 12.530 & $0.092 \sim 0.187$ & 0.006 & 0.975 & 1.361 \\
\hline & Hip abduction & 0.008 & 0.001 & 0.503 & 5.461 & $0.002 \sim 0.014$ & 0.032 & & 1.361 \\
\hline \multirow[t]{3}{*}{3} & Hip extensor & 0.146 & 0.000 & 1.207 & 2112.106 & $0.145 \sim 0.147$ & $<0.001$ & 1.000 & 1.585 \\
\hline & Hip abduction & 0.009 & 0.000 & 0.590 & 926.772 & $0.009 \sim 0.009$ & 0.001 & & 1.966 \\
\hline & Hip flexion & -0.005 & 0.000 & -0.134 & -245.953 & $-0.005 \sim-0.004$ & 0.003 & & 1.449 \\
\hline \multicolumn{10}{|c|}{ peak KAA as dependent variable } \\
\hline 1 & Hip ER moment before peak KAM & 20.237 & 4.364 & 0.937 & 4.637 & $6.350 \sim 34.125$ & 0.019 & 0.837 & 1.000 \\
\hline \multirow[t]{2}{*}{2} & Hip ER moment before peak KAM & 25.090 & 0.626 & 1.161 & 40.076 & $22.396 \sim 27.783$ & 0.001 & 0.998 & 1.416 \\
\hline & Hip ER-or & -1.857 & 0.130 & -0.414 & -14.298 & $-2.416 \sim-1.298$ & 0.005 & & 1.416 \\
\hline \multirow[t]{3}{*}{3} & Hip ER moment before peak KAM & 26.212 & 0.081 & 1.213 & 323.339 & $25.182 \sim 27.242$ & 0.002 & 1.000 & 3.699 \\
\hline & Hip ER-or & -1.819 & 0.011 & -0.406 & -171.112 & $-1.954 \sim-1.684$ & 0.004 & & 1.478 \\
\hline & Hip adduction & -0.033 & 0.002 & -0.067 & -17.622 & $-0.056 \sim-0.009$ & 0.036 & & 3.750 \\
\hline \multicolumn{10}{|c|}{ Group 3 + group 4 . All male participants } \\
\hline model & independent variables & B & $\mathrm{SE}(\mathrm{B})$ & $\beta$ & $\mathrm{t}$ & $95 \% \mathrm{Cl}$ & $P$ & adjusted $R^{2}$ & VIF \\
\hline \multicolumn{10}{|c|}{ peak KAM as dependent variable } \\
\hline \multicolumn{10}{|c|}{ none } \\
\hline \multicolumn{10}{|c|}{ peak KAA as dependent variable } \\
\hline 1 & $\begin{array}{l}\text { maximal Hip ER moment during } 150 \mathrm{~ms} \\
\text { after IGC }\end{array}$ & 19.134 & 2.956 & 0.926 & 6.473 & $12.144 \sim 26.124$ & $<0.001$ & 0.836 & 1.000 \\
\hline
\end{tabular}

Multiple regression analysis was performed with peak KAM and peak KAA as dependent variables, and with demographics and parameters from motion analysis as independent variables. Selection of modeling was done using stepwise method. $B$, partial regression coefficient; $S E$, standard error; $\beta$, standardized partial regression coefficient; $t, t$-ratio; $95 \% \mathrm{Cl}, 95 \%$ confidence interval; $P, P$-value; $R^{2}$, coefficient of determination; VIF, variance inflation factor; IR, internal rotation; ER, external rotation; KAM, knee abduction moment; KAA, knee abduction angle; ER-or, external rotator; IGC, initial ground contact.

independent variables of groups 1 and 2 or groups 3 and 4. In all female participants $\langle$ Table $5>$, independent predictors for peak KAM were ER moment before detecting peak KAM, extension-ROM, extensor strength, IR+ER, and body mass index. An independent predictor for peak KAA was IR angle during $150 \mathrm{~ms}$ after IGC. In all male participants $<$ Table $6>$, there was no independent predictor for peak KAM. An independent predictor for peak KAA was maximal ER moment during $150 \mathrm{~ms}$ after IGC. 


\section{Discussion}

This study is the first to investigate females and males separately based on their hip rotation ROM to elucidate the effect of hip rotation range on knee abduction biomechanics during double-legged drop-landing. Because hip muscle strength may contribute to the frontal-plane knee control during dynamic movements [12], this study matched hip muscle strength between the same sex groups to examine accurate influence of hip rotation range in the same sex. Non-contact ACL injuries typically occur within the first 100 ms after IGC $[9,35]$. Thus, it is important to gain an understanding of the influence of physical abilities including muscular strength on the lower extremity biomechanics during movement tasks, such as the peak KAM and KAA during landing. The present results indicate sex-specific hip strategies based on hip rotation range that may contribute to peak KAM and peak KAA control during double-legged drop landing.

From multiple regression analysis, the males with ER>IR (group 4) could use hip extensor and ER-or to control peak KAM and peak KAA, respectively, during double-legged drop landing. In group 4 peak KAA was also associated with maximal ER moment before detecting peak KAM. The hip tends to collapse into adduction and internal rotation as the hip flexes during weight bearing. This three-dimensional motion is commonly observed during the weight acceptance phase of landing from a jump. In the sagittal plane, the gluteus maximus can serve to diminish the knee loading by decreasing the need for compensatory quadriceps action to absorb impact forces [36]. While the gluteus maximus works as the most powerful ER-or [37], the upper portion of the gluteus maximus can abduct the hip with activation pattern similar to that of the gluteus medius [38]. In the frontal plane, those hip abductors generate abduction torque to counteract the adduction torque produced by body weight and its larger external moment arm acting at the hip [13]. In the transverse plane, the abductor and ER-or act together to control hip and pelvis motion [13]. The gluteus maximus is most highly activated during double-legged sagittal plane landings whereas the gluteus medius activity is highest during single-and double-legged sagittal plane hurdle hops [39]. As a result, the gluteus maximus together with other abductor muscles can provide three-dimensional stability of the hip by resisting the motions of flexion, adduction, and IR [13]. In spite of no data on individual activity of hip musculature, the gluteus maximus most likely controlled peak KAM and peak KAA as the main extensor and ER-or, respectively, during double-legged drop landing in group 4.

Compared with group 4, the males with IR $>$ ER (group 3 ) employed a different landing strategy to control knee abduction biomechanics. In group 3 there were association of peak KAM with ER-ROM and adductor strength and association of peak KAA with ER moment and IR angle during $150 \mathrm{~ms}$ after IGC. Multiple regression analysis with the data from all male participants demonstrated association of peak KAA with maximal ER moment during $150 \mathrm{~ms}$ after IGC and no association between peak KAM and independent variables. This suggests that hip rotation range should be considered in studies on the frontal-plane knee control during landing. Based on a three-dimensional computer model, increased femoral anteversion can decrease the abduction moment arm of abductor [40]. Since maximal ER and IR angles during landing after IGC were similar between groups 3 and 4 , differences in hip rotation range may change the position of the femur relative to the pelvis, thus potentially altering the length, tension, and orientation of the muscles, resulting in changes in hip muscle functions. Gluteal muscles can change their functions depending on the hip position [41]. The posterior fibers of the gluteus maximus that can work as adductor in combination with other adductor muscles [37] may be used to control peak KAM, whereas the gluteus maximus with assistance of other abductors could adjust ER moment and maximal IR angle in the transverse plane during double-legged drop-landing in group 3.

In the females with ER>IR (group 2) peak KAM was associated with maximal ER moment before detecting peak KAM and ER-or strength. Association was also found between peak KAA and maximal hip abduction moment before detecting peak KAM. In group 2, therefore, the gluteus maximus in combination with other abductors may work to adjust ER moment and abduction moment for peak KAM 
and peak KAA control, respectively. In the females with IR $>$ ER (group 1) there was association between peak KAM and maximal hip abduction moment before detecting peak KAM. The gluteus maximus together with other abductors might serve as abductor to adjust hip abduction moment during landing in group 1. However, no association was found between peak KAA and independent variables. From multiple regression analysis with the data from all female participants, independent variables associated with peak KAM and peak KAA were found different from the result of group 1 or 2 . Therefore, hip rotation range should be mindful for studies of females on the frontal-plane knee control during landing.

Comparison between groups 2 and 4 or between groups 1 and 3 highlights sex difference in controlling the frontalplane knee biomechanics during landing based on hip rotation range. Because IR-ER of group 2 was greater than that of group 4 , increased femoral anteversion in group 2 could affect the length, tension, and orientation of the hip musculature and the internal-moment arms of the muscle. The females in group 1 displayed weaker strength of ER-or than the males in group 3. During an anticipated single leg drop landing, females with greater ER-or strength can attenuate vertical ground reaction force more effectively and exhibit smaller knee valgus moments than individuals with lower ER-or strength [42]. Weaker ER-or strength in group 1 compared with group 3 may contribute to altered hip strategy for the frontal-plane knee control.

IR-ER was the greatest in group 1 of the four groups. An increase in femoral anteversion demonstrates positive relationship with knee abduction excursion during landing and negative relationship with knee adduction excursion during landing [11]. In addition, activation of the gluteal muscles is dependent on the length-tension curve where the gluteal muscles may have the greatest activation when they are close to their ideal length [13]. Collectively, the greatest femoral anteversion in group 1 might cause deficits in proximal hip control for knee abduction control during landing. Because males have longitudinal increases in hip strength during adolescent growth in contrast to no strength changes in females, males have greater overall hip strength compared with females [43] as shown in Table 1. Thus, weakness or inefficiency of hip musculature in the females would play another role in the altered hip strategy for knee control. A variety of hip-focused neuromuscular exercise interventions have attempted to gain effective activation of the gluteus medius and the gluteus maximus. Such programs consistently produce improved measures of hip extensor, abductor, and ER-or strength, which can translate to improved biomechanics at the hip and knee during athletic activities in females as a whole [13]. This study may provide additional information on the effect of hip rotation range for refinement of hip-focused interventions.

This study has some limitations. First, the sample size is small. It could be difficult to recruit more females with $E R>I R$ and males with IR>ER because females and males commonly display the opposite difference of hip rotation [16]. Further, the small sample size may cause some selection bias. Second, there are no electromyographic data to examine the relationship between the present results and muscle recruitment of the prime movers at hip joint. Third, the present results are limited to observations during double-legged drop-landing. Generalizations of results from landing-only studies are unlikely to be made with landing followed-by-subsequentactivity studies and other sport-related movements [44]. Last, the present study showed no data on femoral anteversion of each participant, although IR and ER are significantly correlated with radiographic measures of femoral anteversion [15].

\section{Conclusions}

Hip rotation range may affect hip strategy for knee abduction control during double-legged drop-landing, resulting in sex difference in hip-knee coupling in the frontal and transverse planes. Our findings likely give an additional insight into the link between the proximal hip control and the frontal-plane knee loading based on hip rotation range.

\section{Acknowledgments}

This study was supported in part by Japanese Society of Physical Fitness and Sports Medicine (Grant number: 2016- 
H28-30). The authors would like to thank all the participants in this study. The authors would also like to thank Drs. Noriaki Ichihashi and Shinichi Yoshiya for their valuable support to this study.

\section{Conflicts of Interest}

The authors declare no conflict of interest.

\section{References}

1. Agel J, Klossner D. Epidemiologic review of collegiate ACL injury rates across 14 sports: National collegiate athletic association injury surveillance system data 2004-05 through 2011-12. Br J Sports Med. 2014; 48(7):560-560.

2. Hewett TE, Myer GD, Ford KR, et al. Biomechanical measures of neuromuscular control and valgus loading of the knee predict anterior cruciate ligament injury risk in female athletes: a prospective study. Am J Sports Med. 2005; 33(4):492-501.

3. Ford KR, Myer GD, Toms HE, Hewett TE. Gender differences in the kinematics of unanticipated cutting in young athletes. Med Sci Sports. 2005; 37(1):124-129.

4. Olsen OE, Myklebust G, Engebretsen L, Bahr R. Injury mechanisms for anterior cruciate ligament injuries in team handball: a systematic video analysis. Am J Sports Med. 2004; 32(4):1002-1012.

5. Reiman MP, Bolgla LA, Lorenz D. Hip functions influence on knee dysfunction: a proximal link to a distal problem. J Sport Rehabil. 2009; 18(1):33-46.

6. Imwalle LE, Myer GD, Ford KR, Hewett TE. Relationship between hip and knee kinematics in athletic women during cutting maneuvers: a possible link to noncontact anterior cruciate ligament injury and prevention. J Strength Cond Res. 2009; 23(8):2223-2230.

7. Mendiguchia J, Ford KR, Quatman CE, Alentorn-Geli E, Hewett TE. Sex differences in proximal control of the knee joint. Sports Med. 2011; 41(7):541-557.

8. Paterno MV, Schmitt LC, Ford KR, et al. Biomechanical measures during landing and postural stability predict second anterior cruciate ligament injury after anterior cruciate ligament reconstruction and return to sport.
Am J Sports Med. 2010; 38(10):1968-1978.

9. Krosshaug T, Nakamae A, Boden BP, et al. Mechanisms of anterior cruciate ligament injury in basketball: video analysis of 39 cases. Am J Sports Med. 2007; 35(3):359367.

10. Khamis S, Yizhar Z. Effect of feet hyperpronation on pelvic alignment in a standing position. Gait Posture. 2007; 25(1):127-134.

11. Howard JS, Fazio MA, Mattacola CG, Uhl TL, Jacobs CA. Structure, sex, and strength and knee and hip kinematics during landing. J Athl Train. 2011; 46(4):376-385.

12. Myer GD, Chu DA, Brent JL, Hewett TE. Trunk and hip control neuromuscular training for the prevention of knee joint injury. Clin Sports Med. 2008; 27(3):425-448.

13. Ford KR, Nguyen AD, Dischiavi SL, Hegedus EJ, Zuk EF, Taylor JB. An evidence-based review of hip-focused neuromuscular exercise interventions to address dynamic lower extremity valgus. Open Access J Sports Med. 2015; 6:291-303.

14. Fan L, Copple TJ, Tritsch AJ, Shultz SJ. Clinical and instrumented measurements of hip laxity and their associations with knee laxity and general joint laxity. J Athl Train. 2014; 49(5):590-598.

15. Kozic S, Gulan G, Matovinovic D, Nemec B, Sestan B, Ravlic-Gulan J. Femoral anteversion related to side differences in hip rotation: passive rotation in 1,140 children aged 8-9 years. Acta Orthop Scand. 1997; 68(6):533-536.

16. Hogg JA, Schmitz RJ, Nguyen AD, Shultz SJ. Passive hip range-of-motion values across sex and sport. J Athl Train. 2018; 53(6):560-567.

17. Nguyen A, Shultz SJ, Schmitz RJ, Luecht RM, Perrin DH. A preliminary multifactorial approach describing the relationships among lower extremity alignment, hip muscle activation, and lower extremity joint excursion. J Athl Train. 2011; 46(3):246-256.

18. Arnold AS, Komattu AV, Delp SL. Internal rotation gait: a compensatory mechanism to restore abduction capacity decreased by bone deformity. Dev Med Child Neurol. 1997; 39(1):40-44. 
19. Nyland J, Kuzemchek S, Parks M, Caborn DN. Femoral anteversion influences vastus medialis and gluteus medius EMG amplitude: composite hip abductor EMG amplitude ratios during isometric combined hip abduction-external rotation. J Electromyogr Kinesiol. 2004; 14(2):255-261.

20. Hewett TE, Myer GD, Ford KR. Anterior cruciate ligament injuries in female athletes. Part 1. Mechanisms and risk factors. Am J Sports Med. 2006; 34(3):299-311.

21. Svenningsen S, Apalset K, Terjesen T, Anda S. 1989. Regression of femoral anteversion. A prospective study of intoeing children. Acta Orthop Scand. 1989; 60(2):170-173.

22. Upadhyay SS, Burwell RG, Moulton A, Small PG, Wallace WA. Femoral anteversion in healthy children. Application of a new method using ultrasound. J Anat. 1990; 169:49-61.

23. Shultz SJ, Nguyen AD, Schmitz RJ. Differences in lower extremity anatomical and postural characteristics in males and females between maturation groups. J Orthop Sports Phys Ther. 2008; 38(3):137-149.

24. Cohen J. Statistical power analysis for the behavioral sciences (2nd ed.). Lawrence Erlbaum Associates, Hillsdale, New Jersey, USA. 1988, p. 1-579.

25. Ishida T, Koshino Y, Yamanaka M, et al. The effects of a subsequent jump on the knee abduction angle during the early landing phase. BMC Musculoskelet Disord. 2018; 19(1):379.

26. Norkin CC, White DJ. Measurement of joint motion: A guide to goniometry. FA Davis Co., Philadelphia, USA. 2009, p1-448.

27. Thorborg K, Petersen J, Magnusson SP, Holmich P. Clinical assessment of hip strength using a hand-held dynamometer is reliable. Scand J Med Sci Sports 2010: 20(3):493-501.

28. Decker MJ, Torry MR, Wyland DJ, Sterett WI, Richard Steadman J. 2003. Gender differences in lower extremity kinematics, kinetics and energy absorption during landing. Clin Biomech (Bristol, Avon). 2003; 18(7):662669.

29. Blackburn JT, Padua DA. Influence of trunk flexion on hip and knee joint kinematics during a controlled drop landing. Clin Biomech (Bristol, Avon). 2008; 23(3):313319.

30. Leardini A, Biagi F, Merlo A, Belvedere C, Benedetti MG. Multi-segment trunk kinematics during locomotion and elementary exercises. Clin Biomech (Bristol, Avon). $2011 ; 26(6): 562-571$.

31. Itoh H, Takiguchi K, Shibata Y, Okubo S, Yoshiya S, Kuroda R. Correlation between hip function and knee kinematics evaluated by three-dimensional motion analysis during lateral and medial side-hopping. J Phys Ther Sci. 2016; 28(9):2461-2467.

32. Wu G, Siegler S, Allard P, et al. ISB recommendation on definitions of joint coordinate system of various joints for the reporting of human joint motion-part I: ankle, hip, and spine. J Biomech. 2002; 35(4):543-548.

33. Lephart SM, Ferris CM, Riemann BL, Myers JB, Fu FH. Gender differences in strength and lower extremity kinematics during landing. Clin Orthop Relat Res. 2002; 401:162-169.

34. Norcross MF, Lewek MD, Padua DA, Shultz SJ, Weinhold PS, Blackburn JT. Lower extremity energy absorption and biomechanics during landing, part I: sagittalplane energy absorption analyses. J Athl Train. 2013; 48(6):748-756.

35. Koga H, Nakamae A, Shima Y, et al. Mechanisms for noncontact anterior cruciate ligament injuries: knee joint kinematics in 10 injury situations from female team handball and basketball. Am J Sports Med. 2010; 38(11):2218-2225.

36. Pollard CD, Sigward SM, Powers CM. Limited hip and knee flexion during landing is associated with increased frontal plane motion and moments. Clin Biomech. 2010; 25(2):142-146.

37. Neumann DA. Kinesiology of the hip: a focus on muscular actions. J Orthop Sports Phys Ther. 2010; 40(2):82-94.

38. Lyons K, Perry J, Gronley JK, Barnes L, Antonelli D. Timing and relative intensity of hip extensor and abductor muscle action during level and stair ambulation. An EMG study. Phys Ther. 1983; 63(10):1597-1605.

39. Struminger AH, Lewek MD, Goto S, Hibberd E, Blackburn 
JT. Comparison of gluteal and hamstring activation during five commonly used plyometric exercises. Clin Biomech. 2013; 28(7):783-789.

40. Arnold AS, Komattu AV, Delp SL. Internal rotation gait: a compensatory mechanism to restore abduction capacity decreased by bone deformity. Dev Med Child Neurol. 1997; 39(1):40-44.

41. Delp SL, Hess WE, Hungerford DS, Jones LC. Variation of rotation moment arms with hip flexion. J Biomech. 1999; 32(5):493-501.
42. Lawrence RK, Kernozek TW, Miller EJ, Torry MR, Reutmann P. Influences of hip external rotation strength on knee mechanics during single-leg drop landings in females. Clin Biomech. 2008; 23(6):806-813.

43. Brent J, Myer GD, Ford KR, Paterno M, Hewett T. The effect of sex and age on isokinetic hip abduction torques. J Sport Rehabil. 2013; 22(1):41-46.

44. Ambegaonkar JP, Shultz SJ, Perrin DH. A subsequent movement alters lower extremity muscle activity and kinetics in drop jumps vs. drop landings. J Strength Cond Res. 2011; 25(10):2781-2788. 